\title{
Building a Web-based Knowledge Repository on Climate Change to Support Environmental Communities
}

\author{
Arno Scharl ${ }^{1}$ and Albert Weichselbraun ${ }^{2}$ \\ 1 MODUL University Vienna, \\ Am Kahlenberg 1, 1190 Vienna, Austria \\ arno.scharl@modul.ac . at \\ 2 Vienna University of Economics and Business, \\ Augasse 2-6, 1090 Vienna, Austria \\ albert.weichselbraun@wu.ac.at
}

\begin{abstract}
This paper presents the technology base and roadmap of the Climate Change Collaboratory, a Web-based platform that aims to strengthen the relations between scientists, educators, environmental NGOs, policy makers, news media and corporations - stakeholders who recognize the need for adaptation and mitigation, but differ in worldviews, goals and agendas. The collaboratory manages expert knowledge and provides a platform for effective communication and collaboration. It aims to assist networking with leading international organizations, bridges the science-policy gap and promotes rich, self-sustaining community interaction to translate knowledge into coordinated action. Innovative survey instruments in the tradition of "games with a purpose" will create shared meaning through collaborative ontology building and leverage social networking platforms to capture indicators of environmental attitudes, lifestyles and behaviors.
\end{abstract}

\section{Introduction}

Despite credible forecasts and warnings from the scientific community about anthropogenic climate change, greenhouse gas emissions have continued to grow. Scientists studying the issue predict more adverse consequences unless stronger actions are taken $[1,2]$. From the policy-making level down to personal voting and purchasing decisions, however, the observable actions have not been commensurate with the threat of climate change. We remain far short of undertaking the emission reductions that scientists say are required to forestall dangerous interference in the climate system on which our civilization depends [3,4]. Although public concern about climate change has risen dramatically in the past few years, a much smaller percentage is actually taking action $[5,6]$.

Reasons for this discrepancy include: (1) On the micro level, the widespread perception of climate change as a risk that will predominantly impact geographically and temporally distant people and places $[7,8]$; and the lack of personal efficacy (belief that the own actions will make a difference and one's voice will 
be heard), a critical motivating factor in behavioral change [9] that can be supported by electronic networking and Web-based applications to share knowledge and coordinate action. (2) On the meso and macro levels, a gap between policies and research needed to promote and support adaptation, and also mitigation (and their interrelation). The overarching goal of our research is to introduce a Climate Change Collaboratory which builds capacity among policy makers, scientists, educators, environmental NGOs, news media and corporations to close this gap and translate increased awareness into behavioral change on the local, regional, national and international levels.

This paper presents the Media Watch on Climate Change (publicy available at www.ecoresearch.net/climate) as well as the technology extensions necessary to build a Climate Change Collaboratory in an effort to promote environmental action and overcome the inhibiting factors outlined above.

\section{Climate Change Collaboratory}

The Climate Change Collaboratory is an interdisciplinary initiative to encourage and study discourse and critical debate that lead to a shared understanding of climate change issues on all political levels, ranging from inter-individual communication and local communities to global campaigns and treaties.

The project will focus on collaboration between stakeholders often divided by differing world-views, goals and agendas [9]. Environmental Web resources such as documents and best-practice examples are frequently being created through processes of cooperation and social exchange. They depend on and benefit from a synergy of skills, the dynamic maintenance of shared knowledge, flexible and non-hierarchical portfolios of services, and distributed decision-making. The Climate Change Collaboratory recognizes and supports the social construction of meaning via distributed services that aim to improve the quality of decisions, build trust and help resolve conflicts among competing interest. Therefore, it will provide matchmaking services for ad-hoc team composition and a range of Web-enabled communication and collaboration tools.

Currently, the Media Watch on Climate Change (Figure 1) gathers and annotates documents from multiple sources. Data from international associate partners is enriched by third-party material from news media and environmental blogs. Automated text mining methods identify the attention towards current topics, and compute the sentiment of media sources towards these topics automatically. Due to the complex and dynamic nature of the gathered data, there is significant demand for ontologies to structure the domain knowledge. The Climate Change Collaboratory will have access to the required evidence sources for building such ontologies in multiple languages, which are either publicly available or provided by its associate partners.

The Climate Change Collaboratory will extend the functionality of the existing platform by supporting stakeholder communication and collaboration in

a more direct manner. Recent advances in collaborative Web technology are governed by strong network effects and new opportunities to harness collective 


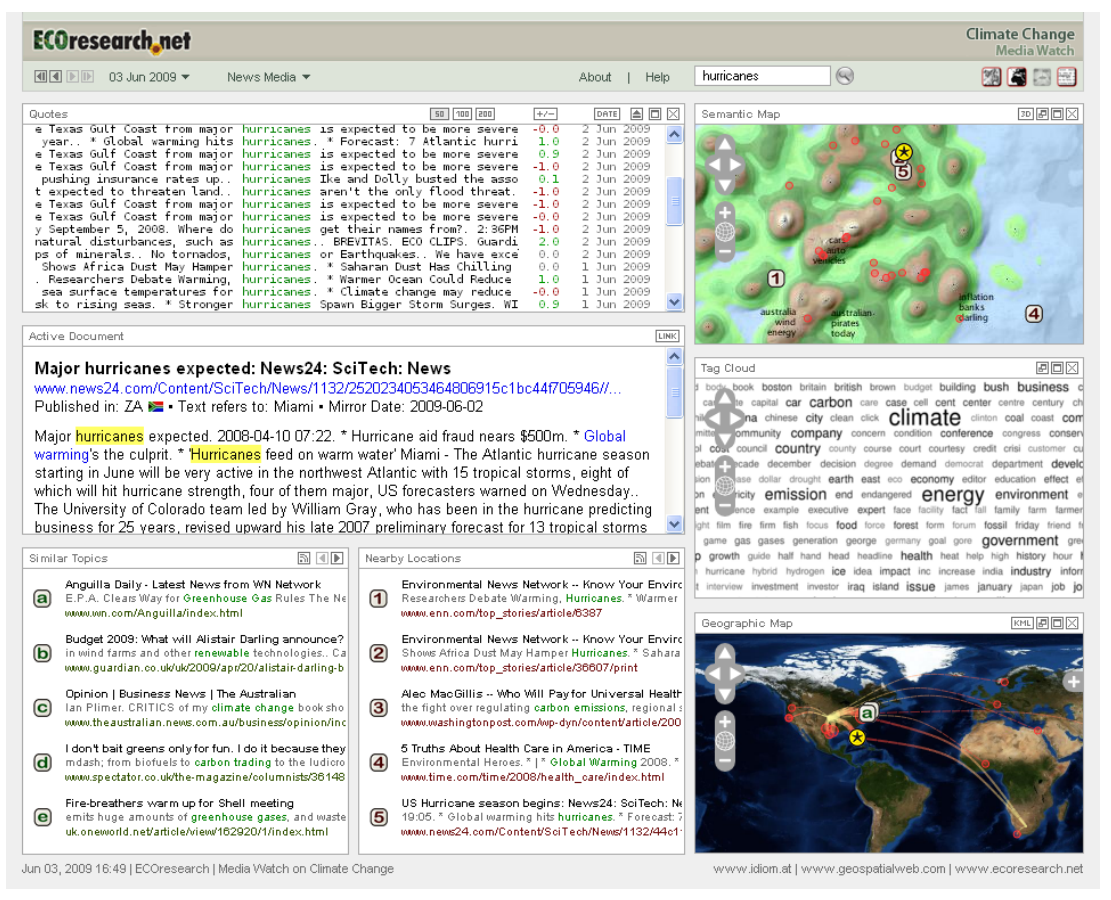

Fig. 1. Media Watch on Climate Change (www.ecoresearch.net/climate)

intelligence. As a result, climate change information spreads rapidly across Web sites, blogs, Wikis, and between users who utilize these services. The Climate Change Collaboratory will capture these rich sources of information to initiate and support stakeholder discourse. We target policy makers and their staff, scientists, educators, environmental NGOs, news media and corporations. These stakeholders will (i) create shared meaning in regards to the underlying concepts, methods, and tools of climate research; (ii) improve their understanding of how others perceive climate change; (iii) build capacity to generate and deliver effective strategies for communicating adaptation and mitigation options, taking into account the dynamic legal and political context; (iv) closing the gap between climate science and public climate literacy; and (v) pool resources with other stakeholders and multiply the impact of their efforts.

Games with purpose $[10,11]$ for evaluation and content creation will be examined as an innovative and cost-effective means to provide indicators of environmental attitudes, lifestyles and behaviors from very large user groups (visitors from the Web sites of core and associate partners, users of Facebook and other networking platforms, etc.). This automated gathering of intercultural data on climate change knowledge will complement conventional social surveys and shed light on the public awareness of climate change, perceived collective and individual threats, support of adaptation and mitigation efforts, and observable behavioral changes. 


\section{Methodology}

The Media Watch on Climate Change benefits from existing resources, both structured and unstructured in nature, and the collective intelligence of the partners' online communities (rather than having to build a new knowledge repository from scratch). The content aggregation and annotation services powering the Media Watch on Climate Change [12] have compiled an archive of contextualized Web documents related to climate change classified by topic and geographic location. This represents a comprehensive source of unstructured data for initializing and maintaining the Climate Change Collaboratory services.

The Climate Change Collaboratory will build on this technology and also considers structured sources such as the GEneral Multilingual Environmental Thesaurus (www.eionet.europa.eu/gemet) which has been developed as an indexing, retrieval and control tool for the European Topic Centre on Catalogue of Data Sources and the European Environment Agency. GEMET was conceived as a general thesaurus, aimed to define a core of general terminology for the environment. Additional evidence sources to be considered are the Global Change Master Directory (gcmd.nasa.gov) of the NASA Goddard Space Flight Center as well as the Semantic Web for Earth and Environmental Terminology (sweet.jpl.nasa.gov/ontology) of the NASA Jet Propulsion Laboratory.

Social Sources will yield demographic data, user-generated content (e.g. reviews or resource annotations), and social relations. All these categories will be used concurrently to gather and validate climate change knowledge. The Climate Change Collaboratory will follow three different approaches to leverage this data: (i) incorporate folksonomies into ontological representations; (ii) use social bookmark services to identify climate change resources for focused crawling, (iii) deploy games with a purpose to interact with users.

Through a combination of social networking sites and addressing users of existing environmental communities, the Climate Change Collaboratory will be able to draw on a huge pool of potential contributors, e.g. more than 250,000 supporters of the WWF Facebook Application and the 40,000 registered WWF Passport users from more than 170 countries, visitors of the NASA Earth Observatory and the NOAA Web Site including its upcoming climate change portal, or the 500 registered users and about 15,000 monthly visitors of the ECOresearch Network.

Utilizing the collective intelligence (integrated analysis of group behavior, preferences and ideas) of these communities will help create shared meaning and encode this meaning in climate change ontologies. Taxonomic relations will be extracted from the aggregated annotations of popular resources by means of statistical clustering [13] and social network analysis [14].

The Climate Change Collaboratory will use bookmarks gained from social networking sites as a source for ontology building via focused crawling. The social bookmarking site Delicious.com, for example, reports more than 35,000 pages tagged with "climate change". The Climate Change Collaboratory will develop tools to capture this knowledge and integrate it into contextualized archives. Assuming that resources tagged by the same user or supplied with identical tags 
are semantically related, one can also identify relevant resources using social data. Social networking platforms and social semantic data embedded in Web documents such as FOAF and XFN will help gathering this data.

A component for semi-automated ontology learning from unstructured text has been continually refined over several years $[15,16]$. It incorporates a range of methods from statistics, artificial intelligence and natural language processing, including co-occurrence analysis, subsumption analysis, link type detection, Hearst patterns, and spreading activation. It addresses the limited availability of expertise and qualified human resources, which is the bottleneck and costdriver in building environmental ontologies. Automated suggestions and intuitive interfaces address this bottleneck by improving the productivity of ontology engineers. Deconstructing the complicated task of ontology building into tiny problems that can be solved by a wider community also offsets the limited availability of domain experts. Web-based interfaces with simple and rapid user feedback mechanisms will generate additional semantic knowledge while browsing the content repository with minimal cognitive effort on behalf of the user.

An integrative view of these data sources will help create a commonly agreed climate change ontology. For non-expert users, however, searching and navigating within ontologies tends to be difficult. The visual interface will address this issue and facilitate intuitive high-level access to the content in the knowledge base.

\section{Outlook and Conclusions}

This paper introduced the Climate Change Collaboratory, a Web-based platform to encourage and study the discourse on climate change, and to create a shared understanding of climate change issues.

The main innovations of the presented collaboratory are (i) providing an integrated view on climate change resources, (ii) interfaces to semi-automatic ontology learning and evaluation standardizing the used terminology between the stakeholders, (iii) evaluating these approaches by applying games with purposes, and (iv) supporting stakeholders in communicating and collaborating by using the Climate Change Collaboratory as a focal point for the exchange and creation of information on global climate change.

Analyzing the discourse and identifying social determinants is essential to obtain an unbiased view on the topic of climate change, which will promote well-informed and responsible policy making and hopefully help to close the gap between scientific studies on the effects of climate change and the decision making of the involved stakeholders.

\section{Acknowledgement}

The project outlined in this paper is funded by the Austrian Climate and Energy Fund within the program line ACRP (Austrian Climate Research Pro-

gram; www.klimafonds.gv.at). The project partners include the Department of New Media Technology (MODUL University Vienna), the Research Institute for 
Computational Methods (Vienna University of Economics and Business), the Wegener Center for Climate and Global Change (University of Graz), and the European Support Center of the Club of Rome.

\section{References}

1. IPCC: Climate Change 2007: Synthesis Report. Intergovernmental Panel on Climate Change, Geneva, Switzerland (2007)

2. Rahmstorf, S., Cazenave, A., Church, J., Hansen, J., Keeling, R., Parker, D., Somerville, R.: Recent climate observations compared to projections. Science 316(5825) (2007) 709

3. Abbasi, D., Speth, J.: Americans and Climate Change: Closing the Gap Between Science and Action. New Haven: Yale School of Forestry \& Environmental Studies (2006)

4. Grossmann, W.D., Steininger, K., Grossmann, I., Magaard, L.: Indicators on economic risk from global climate change. Environmental Science \& Technology 43(16) (2009) 6421-6426

5. EESI: Recent Polling on Public Perceptions of Climate Change. Environmental and Energy Study Institute, Washington, DC (2007)

6. Steininger, K., Weck-Hannemann, H.: Global Environmental Change in Alpine Regions: Recognition, Impact, Adaptation and Mitigation. Edward Elgar Pub, Cheltenham (2002)

7. Cogoy, M., Steininger, K.W.: The Economics of Global Environmental Change: International Cooperation for Sustainability. Edward Elgar, Cheltenham (2007)

8. Marx, S., Weber, E., Orlove, B., Leiserowitz, A., Krantz, D., Roncoli, C., Phillips, J.: Communication and mental processes: Experiential and analytic processing of uncertain climate information. Global Environmental Change 17(1) (2007) 47-58

9. Bowman, T.: Summary Report: A Meeting to Assess Public Attitudes About Climate Change. National Oceanic and Atmospheric Administration, George Mason University Center for Climate Change Communication, Silver Springs (2008)

10. Von Ahn, L.: Games with a purpose. Computer 39(6) (2006) 92-94

11. Rafelsberger, W., Scharl, A.: Games with a purpose for social networking platforms. In: HT '09: Proceedings of the 20th ACM conference on Hypertext and hypermedia, New York, NY, USA, ACM (2009) 193-198

12. Hubmann-Haidvogel, A., Scharl, A., Weichselbraun, A.: Multiple coordinated views for searching and navigating web content repositories. Information Sciences 179(12) (2009) 1813-1821

13. Jäschke, R., Hotho, A., Schmitz, C., Ganter, B., Stumme, G.: Discovering shared conceptualizations in folksonomies. Web Semantics: Science, Services and Agents on the World Wide Web 6(1) (2008) 38-53

14. Knoke, D., Yang, S.: Social Network Analysis. 2nd edn. SAGE Publications

15. Liu, W., Weichselbraun, A., Scharl, A., Chang, E.: Semi-automatic ontology extension using spreading activation. Journal of Universal Knowledge Management 0(1) (2005) 50-58

16. Weichselbraun, A., Wohlgenannt, G., Scharl, A., Granitzer, M., Neidhart, T., Juffinger, A.: Discovery and evaluation of non-taxonomic relations in domain ontologies. International Journal of Metadata, Semantics and Ontologies 4(3) (2009) $212-222$ 